\title{
COVID-19 in adults with congenital heart disease: early morbidity and mortality in two northern Italian specialist centers
}

\author{
Paolo Ferrero, ${ }^{1}$ Massimo Chessa, ${ }^{2}$ Isabelle Piazza, ${ }^{3}$ Caterina Bonino, ${ }^{1}$ Irene Ferrari, ${ }^{1}$ Alessandro Giamberti, ${ }^{4}$ \\ Matteo Ciuffreda, ${ }^{1}$ Konstantinos Dimopoulos ${ }^{5}$ \\ ${ }^{1}$ Cardiology Department, ASST Papa Giovanni XXIII, Bergamo, Italy; ${ }^{2}$ ACHD Unit - Pediatric and Adult Congenital Heart \\ Centre; IRCCS-Policlinico San Donato, San Donato Milanese (MI), Italy; ${ }^{3}$ University of Milan, Italy; ${ }^{4}$ Surgical Congenital \\ Heart Centre; IRCCS-Policlinico San Donato, San Donato Milanese (MI), Italy; ${ }^{5}$ Adult Congenital Heart Centre, Royal Bromp- \\ ton Hospital, and National Heart and Lung Institute, Imperial College, London, UK
}

\begin{abstract}
There is no information on coronavirus disease 2019 (COVID-19)-related outcomes for adult patients with congenital heart disease (ACHD). We aim to assess the clinical burden of COVID-19 on ACHD patients during the initial stages of the pandemic in Europe. ACHD patients followed in 2 tertiary centers in the Lombardy region were screened for COVID-19-related symptoms and complications. 661 patients $(52.3 \%$ male, mean age $34.9 \pm 11.7$ years) with CHD of moderate (58.5\%) or high (32.8\%) complexity were contacted. Of these, 145 (21.9\%) reported typical COVID-19 symptoms, but only $4.8 \%$ required hospitalization. We did not record confirmed COVID-19-related deaths. Patients hospitalized were elderly $(\mathrm{P}=0.03)$, more likely to be receiving diuretics $(\mathrm{P}=0.03)$ or beta-blockers $(\mathrm{P}=0.02)$, and have been admitted over the previous year $(\mathrm{P}=0.003)$. Of the 7 patients hospitalized, 2 had a systemic RV and 2 a Fontan circulation. COVID-19-related morbidity and mortality have been low in ACHD patients 6 weeks from the start of the outbreak, in one of the worse hit European regions.
\end{abstract}

Correspondence: Paolo Ferrero, Cardiology Department, ASST Papa Giovanni XXIII, Piazza OMS 1, 24127 Bergamo, Italy. Tel.: +39.0352673529. E-mail: pferrero@asst-pg23.it Massimo Chessa, ACHD Unit - Pediatric and Adult Congenital Heart Centre; IRCCS-Policlinico San Donato, San Donato Milanese (MI), Italy.

E-mail: massimo.chessa@grupposandonato.it

Key words: Adult congenital heart disease; COVID-19; survey.

Acknowledgments: we thank AICCA-Onlus, the Italian ACHD Patient Association, for its support in promoting the survey.

Contributions: $\mathrm{PF}$ and MC contributed equally as first authors; IP, CB, IF screened the patients and collected the data; $\mathrm{AG}$ and $\mathrm{MC}$ reviewed the final paper; $\mathrm{KD}$ made the statistical analysis and critically reviewed the paper.

Conflict of interests: the authors declare no potential conflict of interests.

Availability of data and materials: data used to support the findings of this study are available from the corresponding author upon request.

Ethics approval and consent to participate: not required.

Received for publication: 24 October 2020.

Accepted for publication: 27 October 2020.

This work is licensed under a Creative Commons Attribution NonCommercial 4.0 License (CC BY-NC 4.0).

${ }^{\circ}$ Copyright: the Author(s), 2021

Licensee PAGEPress, Italy

Italian Journal of Medicine 2021; 15:27-33

doi:10.4081/itjm.2021.1397

\section{Introduction}

Northern Italy has been hit particularly hard by the coronavirus disease 2019 (COVID-19)pandemic, with an exponential increase in the number of patients requiring hospital admission and intensive care, overwhelming the national health system and resulting in significant restrictions for the general population. ${ }^{1,2}$ The region of Lombardy has been the epicenter of this pandemic in Italy. Most hospitals have been transformed into COVID-19 centers, almost exclusively dedicated to the care of infected patients, and a large proportion of the population in this region was exposed to the virus. This was the first major COVID-19 outbreak in Europe.

Preliminary data from China have suggested that patients with chronic heart conditions are at increased risk of complications with the COVID-19 infection..$^{2-4}$ Congenital heart disease (CHD) includes a broad spectrum of chronic cardiovascular conditions and is the most common inborn defect in humans. ${ }^{5}$ Yet, little is known on the impact of COVID-19 on patients with CHD and the measures that such patients should take to minimize the risk of infection and its complications. ${ }^{6}$ Moreover, most elective work, including routine CHD follow-up, has been canceled during this outbreak, both in terms of capacity and to minimize travel for patients and the risk of infection. ${ }^{7-9}$

In most adult CHD (ACHD) centers, support to patients with COVID-19-related (or other) queries is provided by telephone or email. We present COVID- 
19-related data on a large number of ACHD patients followed in 2 tertiary centers in Northern Italy, who were contacted 6 weeks after the beginning of the outbreak in this region. The study aimed to provide an overview of epidemiology, risk management, and prevalence of symptoms suggestive of COVID-19 infection among ACHD patients at the early stages of the pandemic.

\section{Materials and Methods}

ACHD patients from two specialist centers were contacted by phone between $20^{\text {th }}$ March and $8^{\text {th }}$ April 2020. This included: i) all patients followed in the ACHD department of Bergamo for whom valid contact details were available; and ii) patients followed at San Donato Hospital (Milan) who were registered on the AICCA-Onlus (Italian ACHD Patient Association). Patients with minor/simple lesions (e.g., patent foramen ovale, small/repaired patent ductus arteriosus, a small atrial septal defect, or mild valve disease) were excluded. On contacting patients, the following variables were retrieved: date of the last appointment, admission to hospital over the last year, current therapy, COVID-19 symptoms over the previous 6 weeks, social and/or workplace exposure to possible and/or confirmed cases of COVID-19, regular use of personal protection equipment (PPE), and overall risk perception. The latter was qualitatively graded into a poor, medium, and high understanding of the potential risk that COVID-19 poses to their health by the interviewing physician. Patients spontaneously isolating at home, asking for advice on risk minimization and/or implementing spontaneous COVID-related lifestyle changes were considered by the interviewer as having a greater risk perception. Patients with a combination of at least two of the following symptoms in the preceding 6 weeks were recorded as having had typical COVID-19 symptoms: fever, cough, myalgia, gastrointestinal symptoms, anosmia/ageusia. All patients were advised to remain at home, avoid contacts at risk and use PPE when exiting their homes. A dedicated number was also provided to patients, who were invited to notify us of any significant change in their clinical status. Patients with typical mild-tomoderate symptoms were invited to remain at home, liaising with their general practitioner. In case of persisting or worsening respiratory symptoms, patients were invited to attend their local emergency department for further investigations.

\section{Statistical analysis}

Data were summarized as mean \pm standard deviation or median [range or interquartile range (IQR)] for continuous variables, and number (\%) for categorical variables. Comparison of continuous variables between groups was performed using non-parametric Wilcoxon rank-sum test or Kruscal-Wallis test, and categorical variables were compared using Fisher's exact test or chi-square test. For all analyses, a two-tailed P-value $<0.05$ was used as the criterion for statistical significance. Statistical analyses were performed using $\mathrm{R}$ version 3.6.2 (The $\mathrm{R}$ Foundation for Statistical Computing).

\section{Results \\ Population}

A total of 661 patients were contacted. Of these, $495(74.9 \%)$ were followed in Bergamo and 166 $(25.1 \%)$ in San Donato, Milan. The mean age was $34.9 \pm 11.7$ years, and $345(52.3 \%)$ were male. While the vast majority $(\mathrm{n}=477,72.4 \%)$ resided in the Lombardy region, mainly in the cities of Bergamo $(\mathrm{n}=194$, $30.6 \%)$ and Milan ( $\mathrm{n}=84,13.2 \%)$, there were patients from most Italian regions (Figures 1A and 2), predominantly the north.

Most patients had CHD of moderate $(58.5 \%)$ or high complexity (32.8\%). Common diagnoses included tetralogy of Fallot ( $\mathrm{n}=146,22.1 \%$ ), followed by patients with a Fontan-type circulation ( $\mathrm{n}=70,10.6 \%)$, left ventricular outflow tract (LVOT)/aortic valve disease $(\mathrm{n}=67,10.1 \%)$, and atrioventricular septal defects $(\mathrm{n}=60,9.1 \%)($ Table 1). A systemic right ventricle was present in $34(5.1 \%)$ patients with transposition of the great arteries (TGA) after atrial switch repair or congenitally corrected TGA. There were 23 (3.5\%) patients with complex cyanotic heart defects. At the time of contact, $3(0.5 \%)$ patients were pregnant.

Overall, 96 (15.2\%) were taking an ACE inhibitor (ACEi) or angiotensin receptor blocker (ARBs), 257 (40.8\%) a beta-blocker, and $143(23.0 \%)$ were on a diuretic. Many were on anticoagulants $(\mathrm{n}=144,22.9 \%)$ or antiplatelet therapy $(\mathrm{n}=99,15.7 \%)$, and 52 were on antiarrhythmics (7.8\%). There were 24 (3.8\%) patients on pulmonary arterial hypertension therapy. Less than half of patients $(42.5 \%)$ had received a flu jab during this season (Table 2).

One out of five patients (18.3\%) had required hospital admission in the previous year for a variety of reasons, including surgery $(25.0 \%)$, cardiac catheterization or intervention (20.8\%), heart failure $(9.2 \%)$, or arrhythmia $(19.2 \%)$. Three patients were in the first trimester of pregnancy, and another patient had recently undergone heart transplantation; all of these were socially isolated and completely asymptomatic.

\section{Symptoms of COVID-19 and contacts}

A total of $145(21.9 \%)$ patients described symptoms typical of COVID-19 infection on an 
average of 23.0 [IQR 10.2-34.0] days before the telephone contact, and other $2(0.3 \%)$ patients soon after our telephone communication. The symptoms persisted on average 7.0 [IQR 5.0-9.0] days and included fever (reported in $16.8 \%$ of the entire population), cough (15.1\%), myalgia (9.7\%), rhinitis $(9.8 \%)$, and fatigue $(8.6 \%$, Figure 2$)$. Patients with typical symptoms were more likely to reside in the region of Lombardy $(84.1 \%, \mathrm{P}=0.0003)$, be living with their family $(44.1 \%, \mathrm{P}=0.005)$, receiving amiodarone $(1.7 \%, \mathrm{P}=0.007)$, and have been in contact with a possible or confirmed COVID-19 case $(78.6 \%, \mathrm{P}<0.0001)$. Indeed, a total of $270(40.8 \%)$ patients reported at least one contact with a possible $(\mathrm{n}=223,33.7 \%)$ or confirmed $(\mathrm{n}=47,7.1 \%)$ COVID19 case on average 20.0 [IQR 14.0-30.0] days prior to the telephone conversation. This included 114 (78.6\%) patients with typical symptoms, in whom contacts had occurred either within their families (69.5\%) or in-hospital (30.5\%).

A COVID-19 swab was performed in 14 patients ( $2.1 \%$ of the overall cohort, $8.3 \%$ of those with typical symptoms) and was positive in 12 patients $(1.8 \%$ of the overall population, $7.6 \%$ of those with typical symptoms). Positive patients were elderly ( $\mathrm{P}=0.01$ ) and more likely to have a Fontan or TGA with atrial switch diagnosis $(\mathrm{P}=0.003)$ (Table 2).

\section{All patients}
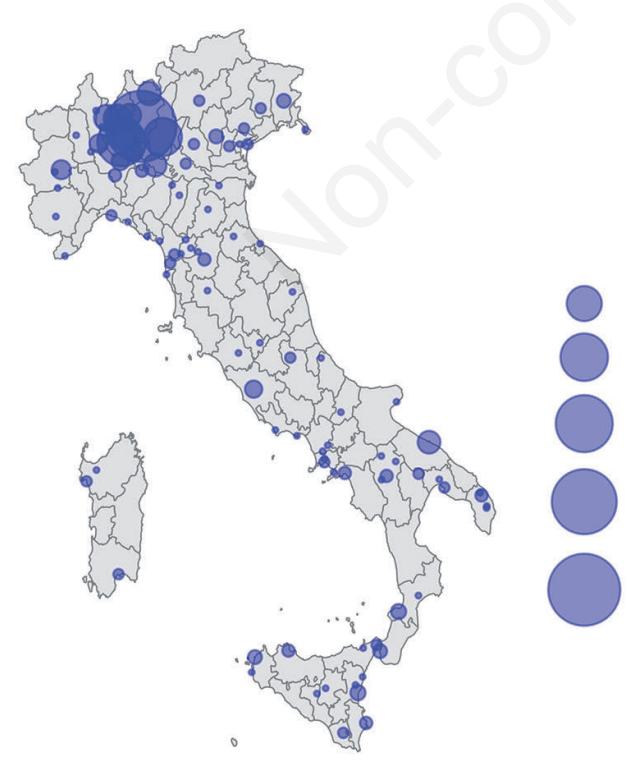

A

Figure 1. In A) distribution of patients according to the place of residence in Italy. Most patients resided in northern Italy. In B) patients who developed typical symptoms.
Table 1. Prevalence of anatomic categories.

\begin{tabular}{lcc}
\hline TOF/PA-VSD & $\mathbf{N}$ & $\mathbf{\%}$ \\
\hline Single ventricle/ Fontan & 146 & 22 \\
\hline AVSD/AVSD-TOF & 70 & 10.6 \\
\hline Aortic Coarctation & 60 & 9.1 \\
\hline Aortic valve disease & 48 & 7.2 \\
\hline ASD/PAPVD & 47 & 7.1 \\
\hline PS/PA-IVS & 45 & 6.8 \\
\hline VSD & 44 & 6.1 \\
\hline TGA complex & 35 & 5.2 \\
\hline Ebstein & 34 & 5.1 \\
\hline STGA/Atrial switch & 24 & 3.6 \\
\hline Complex-cyanotic & 23 & 3.4 \\
\hline STGA/Arterial switch & 23 & 3.4 \\
\hline Aortic valve disease/LVOTO & 21 & 3.1 \\
\hline Truncus & 20 & 3 \\
\hline PDA & 11 & 1.6 \\
\hline ALCAPA & 6 & 0.9 \\
\hline
\end{tabular}

TOF/PA-VSD, tetralogy of Fallot/pulmonary atresia with ventricular septal defect; AVSD, atrioventricular septal defect; ASD, atrial septal defect; STGA, simple (isolated) transposition of great arteries; IVS, intact ventricular septum; PDA, patent ductus arteriosus; LVOTO, left ventricular outflow obstruction; ALCAPA, anomalous left coronary artery from pulmonary artery.

\section{Patients with typical symptoms}

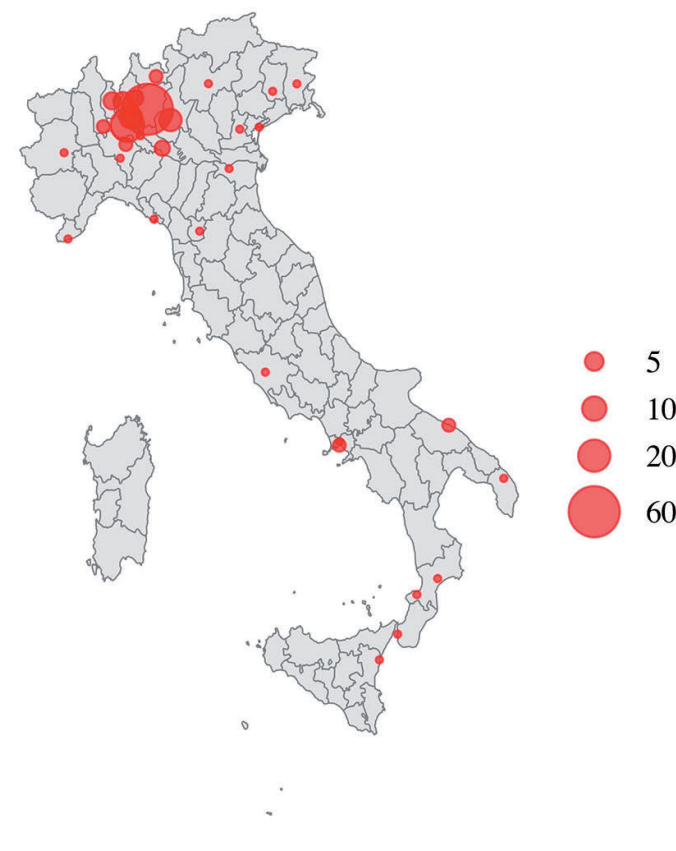




\section{Hospital admissions and mortality}

Hospital admission was required in 7 patients $(1.1 \%$ of the total, $4.8 \%$ of those with typical symptoms) on an average of [range] 8.5 [8.0-15.0] days. Two patients were diagnosed with COVID-19 during hospital admission for an operation at the time of lock-down. The median age of hospitalized patients was 46.8 [4347.5] years and was significantly higher to those who were not hospitalized (32.6 [25.5-42] years, $\mathrm{P}=0.03$ ). These patients were also more likely to be receiving diuretics $(57.1 \%$ versus $19.6 \%, \mathrm{P}=0.03)$ or betablockers $(85.7 \%$ versus $40.3 \%, \mathrm{P}=0.02)$ and have been admitted over the previous year $(71.4 \%$ versus $17.7 \%$, $\mathrm{P}=0.003)$. Their background diagnosis was a Fontantype operation in 2 patients, atrial switch repair of TGA in 2 patients, repaired AVSD in 2 patients and LVOT disease in 1 patient $(\mathrm{P}=0.009$ for atrial switch or Fontan patients versus the remainder). There was, however, no

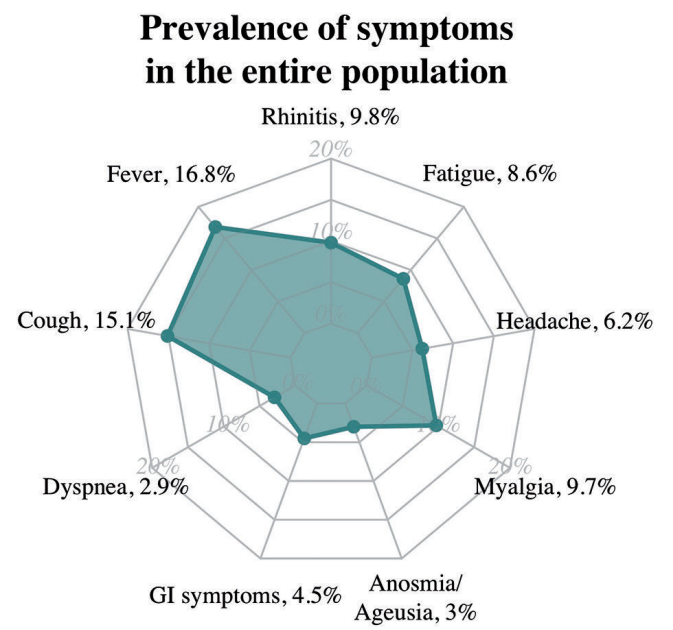

Figure 2. Radar plot depicting the proportion of patients who developed each of the COVID-19 symptoms: fever and cough were most commonly reported.

Table 2. Characteristics of the overall population and patients with and without typical COVID-19 symptoms.

\begin{tabular}{|c|c|c|c|c|}
\hline & $N=661$ & $\begin{array}{l}\text { Typical symptoms } \\
\qquad \mathbf{N}=145\end{array}$ & $\begin{array}{c}\text { Absence of } \\
\text { typical symptoms } \\
\mathrm{N}=\mathbf{5 1 6}\end{array}$ & $\mathbf{P}$ \\
\hline Male, N (\%) & $345(52)$ & $82(56)$ & $263(51.1)$ & 0.28 \\
\hline Age, median [IQR] & $34.5[13.8-75.6]$ & $37.9[27.2-48.8]$ & $33.3[25.8-40.6]$ & 0.06 \\
\hline Patients resident in Lombardy, N (\%) & $477(72.4)$ & $122(84.1)$ & $23(15.9)$ & 0.0003 \\
\hline Living with parents, N (\%) & $34.9 \pm 11.7$ & $64(44.1)$ & $297(57.6)$ & 0.005 \\
\hline Time to last appointment (months), median [IQR] & $6.3[0.16-74]$ & $5.5[3.3-10.4]$ & $7.2[3.5-12.2]$ & 0.37 \\
\hline Admission in the last year, $\mathrm{N}(\%)$ & $361(54.6)$ & $25(17.2)$ & $96(18.6)$ & 0.79 \\
\hline $\begin{array}{l}\text { Bethesda classification } \\
\text { Simple, N (\%) } \\
\text { Moderate complexity, N (\%) } \\
\text { High complexity, N (\%) }\end{array}$ & $\begin{array}{c}57(8.6) \\
387(58.5) \\
217(32.8)\end{array}$ & $\begin{array}{l}14(9.7) \\
83(57.2) \\
48(33.1)\end{array}$ & $\begin{array}{c}43(8.3) \\
304(58.9) \\
169(32.8)\end{array}$ & 0.86 \\
\hline Pregnant, N (\%) & $3(0.5)$ & $1(0.7)$ & $2(0.4)$ & \\
\hline $\begin{array}{l}\text { Therapy } \\
\text { Beta-blocker, N (\%) } \\
\text { Loop diuretic, N (\%) } \\
\text { Aldosterone antagonist, N (\%) } \\
\text { ACEi/ ARB, N (\%) } \\
\text { Amiodaron, N (\%) } \\
\text { Other antiarrhythmic, N (\%) } \\
\text { Anticoagulant, N (\%) } \\
\text { Antiplatelet, N (\%) } \\
\text { PAH, N (\%) }\end{array}$ & $\begin{array}{c}257(40.8) \\
126(20) \\
79(12.6) \\
96(15.2) \\
17(2.7) \\
30(4.8) \\
144(22.9) \\
99(15.7) \\
24(3.8)\end{array}$ & $\begin{array}{c}59(40.7) \\
101(20.8) \\
22(15.2) \\
21(14.5) \\
9(6.2) \\
2(1.4) \\
33(22.8) \\
23(15.9) \\
7(4.8)\end{array}$ & $\begin{array}{c}198(40.8) \\
25(17.2) \\
57(11.8) \\
75(15.4) \\
8(1.7) \\
28(5.8) \\
111(22.9) \\
76(15.7) \\
17(3.5)\end{array}$ & $\begin{array}{c}1 \\
0.47 \\
0.34 \\
0.88 \\
0.007 \\
0.05 \\
1 \\
1 \\
0.67\end{array}$ \\
\hline COVID-19 swab, N (\%) & $14(2.1)$ & $14(100)$ & 0 & \\
\hline Flu immunization, N (\%) & $280(42.8)$ & $65(44.8)$ & $215(41.8)$ & 0.58 \\
\hline Duration of symptoms (days), median [IQR] & $7.0[5.0-9.0]$ & & & \\
\hline Contact with possible/confirmed case, N (\%) & $270(40.8)$ & $114(78.6)$ & $34(0.7)$ & $<0.0001$ \\
\hline Patient currently working, N (\%) & $59(8.9)$ & $13(9)$ & $44(8.5)$ & 1 \\
\hline Social distancing, N (\%) & $602(91.1)$ & $130(90)$ & $472(91.5)$ & 0.13 \\
\hline Regular PPE use, N (\%) & $437(66.1)$ & $107(73.8)$ & $330(64)$ & 0.22 \\
\hline $\begin{array}{l}\text { Risk perception } \\
\text { Low, N (\%) } \\
\text { Intermediate, N (\%) } \\
\text { High, N (\%) }\end{array}$ & $\begin{array}{c}77(11.6) \\
343(52) \\
241(36.4)\end{array}$ & $\begin{array}{l}16(11.1) \\
83(57.2) \\
46(31.7)\end{array}$ & $\begin{array}{c}59(11.5) \\
260(50.5) \\
197(38)\end{array}$ & 0.33 \\
\hline
\end{tabular}

IQR, interquartile range; ACEi, angiotensin-converting enzyme inhibitors; ARB, angiotensin receptor blocker; PAH, pulmonary arterial hypertension; PPE, personal protection equipment. 
relation between anatomic complexity according to the Bethesda classification and hospitalization $(\mathrm{P}=0.32) .{ }^{10}$ There was 1 death in a 23-year-old woman with Down syndrome and a repaired AVSD, who died of lung sepsis causing multiorgan failure. Nasopharyngeal swab had not been performed. Two patients with more severe presentation had a high body mass index (30 and 32, respectively) and comorbidities: 1 patient with diabetes mellitus and chronic heart failure, and 1 with systemic hypertension. Only one patient required admission to intensive care. COVID-19 therapy was given to 13 patients $(8.7 \%$ of patients with typical symptoms, Table 3).

\section{Perception of risk and preventive measures}

The majority of patients were deemed to have a moderate $(52 \%)$ or high $(36.4 \%)$ perception of the risk posed by COVID-19 on their health. Risk perception was higher in patients on treatment with a diuretic $(\mathrm{P}=0.0005)$, those living with their family $(\mathrm{P}=0.04)$ and patients with a more recent ACHD follow appointment $(\mathrm{P}=0.001)$, while there was no relation to anatomic severity $(\mathrm{P}=0.49)$. The vast majority $(\mathrm{n}=602$, $91.1 \%$ ) had been practicing social distancing for 21.0 [15.0-30.0] days on an average before the call. Moreover, 437 (66.1\%) patients were using personal protective equipment (such as facemasks and gloves) in their everyday activities outside their homes. The median number of exits per week from home was 1.0 [0.0-2.0], with $15.7 \%$ exiting their homes $\geq 3$ times per week and $38.7 \%$ none. Most patients who did exit their homes used either their own means of transport
$(37.9 \%)$ or walked $(56.8 \%)$, with few using public transport (1.0\%). A minority of patients were currently going to work ( $\mathrm{n}=59,8.9 \%)$, with $25(3.8 \%)$ holding an essential/key position, hence exiting their home more often than the remainder (median 7 versus 1/per week, $\mathrm{P}<0.0001)$. Most patients $(\mathrm{n}=140,95.2 \%)$ on vitamin $\mathrm{K}$ antagonists who required INR checks continued to attend anticoagulation clinics, while a minority ( $n=7,4.8 \%$ ) had been switched to low molecular weight heparin.

\section{Discussion}

Even though a large number of ACHD individuals experienced symptoms typical of COVID-19 infection over the last 6 weeks, few patients required hospital admission, and only one needed intensive care. One patient died of sepsis but was not tested for COVID19 , and very few patients with typical symptoms were swabbed. Patients with systemic RVs after atrial switch for TGA, and those with univentricular hearts, may be more prone to complications leading to hospitalization, even though more data are needed to confirm this trend. High levels of awareness of the virus's risk, and adherence to preventive measures may explain the low COVID-19-related morbidity and mortality in this population.

Though young, the described ACHD population consisted mainly of patients with congenital defects of moderate or severe complexity. A quarter of patients were receiving diuretics, and more than a third were taking beta-blockers. Despite the complexity of the

Table 3. Characteristics of hospitalized versus non-hospitalized patients.

\begin{tabular}{|c|c|c|c|}
\hline & $\begin{array}{l}\text { Hospitalized for COVID-19 } \\
\qquad \mathrm{N}=7\end{array}$ & $\begin{array}{l}\text { Non-hospitalized } \\
\qquad \mathrm{N}=654\end{array}$ & $\mathbf{P}$ \\
\hline Male, N (\%) & $3(42.9)$ & $342(52.4)$ & 0.90 \\
\hline Age, median [IQR] & $46.8[43-47.5]$ & $32.6[25.5-42]$ & 0.034 \\
\hline Admission in the last year, N (\%) & $5(71.4)$ & $116(17.7)$ & 0.002 \\
\hline $\begin{array}{l}\text { Bethesda classification } \\
\text { Simple, N (\%) } \\
\text { Moderate complexity, N (\%) } \\
\text { High complexity, N (\%) }\end{array}$ & $\begin{array}{c}0(0) \\
3(42.9) \\
4(57.1)\end{array}$ & $\begin{array}{c}57(8.7) \\
384(58.7) \\
213(32.6)\end{array}$ & 0.33 \\
\hline $\begin{array}{l}\text { Therapy } \\
\text { Beta-blocker, N (\%) } \\
\text { Loop diuretic, N (\%) } \\
\text { Aldosterone antagonist, N (\%) } \\
\text { ACEi/ARB, N (\%) } \\
\text { Amiodarone, N (\%) } \\
\text { Other antiarrhythmic, N (\%) } \\
\text { Anticoagulant, N (\%) } \\
\text { Antiplatelet, N (\%) } \\
\text { PAH, N (\%) }\end{array}$ & $\begin{array}{l}6(85.7) \\
4(57.1) \\
1(14.3) \\
2(28.6) \\
1(14.3) \\
1(14.3) \\
4(57.1) \\
0(0) \\
1(14.3)\end{array}$ & $\begin{array}{c}251(40.3) \\
122(19.6) \\
78(12.5) \\
94(15.1) \\
16(2.6) \\
29(4.7) \\
140(22.5) \\
99(15.9) \\
23(3.7)\end{array}$ & $\begin{array}{l}0.04 \\
0.03 \\
1 \\
0.64 \\
0.46 \\
0.76 \\
0.08 \\
0.53 \\
0.64\end{array}$ \\
\hline Flu immunization, N (\%) & $5(71.4)$ & $275(42.2)$ & 0.24 \\
\hline Specific COVID-19 therapy, N (\%) & $6(85.7)$ & $7(4.9)$ & $<0.0001$ \\
\hline
\end{tabular}

$\mathrm{IQR}$, interquartile range; ACEi, angiotensin-converting enzyme inhibitors; ARB, angiotensin receptor blocker; PAH, pulmonary arterial hypertension. 
underlying disease and the fact that one out of five patients in our cohort developed typical COVID-19 symptoms over the 6 weeks before us contacting them, very few patients reported significant complications from the infection. One patient with Down syndrome died in another hospital from lung sepsis, but without confirmation of COVID-19 infection. Patients with Down syndrome are prone to complications from respiratory or other infections, and comorbidities (e.g., obesity, sleep apnea, airways disease, metabolic abnormalities) are common., ${ }^{4,6,11}$ Several patients also required hospitalization, especially old individuals with more advanced disease or comorbidity, including obesity and systemic hypertension.

Despite the fairly encouraging results of our study, caution is needed to avoid complacency. This is the beginning of the COVID outbreak, and despite the alarming number of hospitalizations and deaths in Italy, a minority of the overall population is likely to have, as yet, been exposed. Early introduction of social distancing measures is likely to protect our most vulnerable patients by reducing the peak of the outbreak and allowing hospitals to provide care to all who need it, young and old ACHD patients, with simple or complex defects. The high levels of risk perception and adherence to preventive measures recorded in our population may explain the low early morbidity and mortality described. Indeed, most patients were practicing social distancing at the time of telephone contact, only occasionally leaving their homes, mainly working from home. Many also used PPE when exiting their homes, even though there is still debate on the efficacy of PPE in the general population. ${ }^{12}$ It is alarming that, despite strict hospital protocols, 2 patients had been infected during hospital admission for planned cardiac surgery, demonstrating how difficult it is to maintain separate pathways for COVID and non-COVID patients in the hospital. Indeed, most specialist ACHD centers have ceased routine activity, minimizing the risk of exposure to patients through travel and attendance to hospital visits or admissions. ${ }^{7}$ Special pathways should be put in place for ACHD patients needing urgent or emergency intervention, or other in-hospital treatment, carefully weighing the benefits of such treatment against the risk of COVID-19 infection.

The vast majority of our patients with typical symptoms had not been tested for COVID-19, and most of those tested had been positive. This poses a significant dilemma, as it suggests that a large number of COVID-19 cases in the community may be missed, making accurate estimates of COVID-related morbidity and mortality impossible. For this reason, we opted to provide information on patients with typical symptoms, not only those testing positive or hospitalized. Our approach is supported by the fact that most patients with typical symptoms resided in the Lombardy region, where the outbreak had been the strongest. Widespread use of assay testing for COVID-19 antibodies will improve our understanding of the impact of this pandemic and how to protect our population. ${ }^{13}$

Education is a fundamental part of the care of ACHD patients. Patients followed in specialist centers receive life-long expert care and are educated on the potential complications of $\mathrm{CHD}$, including endocarditis, and how to mitigate risk. ${ }^{14,15}$ Thus, it is not surprising that the majority of our patients were aware of the potential risks of COVID-19 and had taken measures to avoid infection. Educational programs, such as webinars and other web-based resources, produced by ACHD experts in collaboration with scientific societies and patient associations, are ideal for providing our patients with up-to-date, ACHD-specific information during this outbreak.

In our cohort, $15.2 \%$ of patients were taking an ACEi or ARB. We found no relation between this medication and the risk of developing typical symptoms or being hospitalized for COVID-19. There is currently insufficient evidence to warrant interruption of such treatments during the COVID-19 outbreak. ${ }^{16}$ Most patients on vitamin $\mathrm{K}$ antagonists were attending anticoagulation clinics, risking exposure to the virus. Preliminary data suggest that non-vitamin $\mathrm{K}$ oral anticoagulants (NOACs) may be a viable option for selected ACHD patients. ${ }^{17}$ Home testing kits are an alternative for patients in whom NOACs are not an option (e.g., those with metallic prosthetic valves). ${ }^{18}$

\section{Limitations of the study}

Our study is greatly limited by the lack of extensive community testing for COVID-19. Thus, we could not confirm how many of our patients with typical symptoms had indeed been exposed to COVID19. These data were collected early in the course of the pandemic, even though the outbreak in Northern Italy has been significant, both in terms of infection rates and mortality. The latter in Italy exceeded the Chinese one, where the pandemic started. Data were collected during the early peak of the pandemic when the healthcare system in northern Italy (and around the world) was unprepared for the scale of this health crisis, and the availability of swabs was limited, reserved mainly for inpatients. On the other hand, patients with mild to moderate symptoms were managed at home, and only severely affected patients were hospitalized. While symptoms are non-specific, the presence of typical symptoms was felt to be an acceptable early approximation of the likelihood of a COVID-19 diagnosis.

With the use of technology, ongoing surveillance is paramount in maintaining constant contact with the patients and their families. 
The assessment of patients' perception of risk was subjective and qualitative, based on their understanding of the risks posed by the pandemic to their health, and active engagement in minimizing risk, predominantly by practicing strict social distancing, which has, nowadays, become one of the pillars of our fight against COVID-19. Categorization of risk was also empirical; yet, in the absence of data, it is the approach adopted by national and international ACHD societies, who have produced lists of conditions that could predispose patients to complications from COVID-19, which are based on expert consensus. Given the importance attributed to these preventive measures in the initial (and later) phases of the pandemic, we felt that patient risk perception, although poorly standardized, can play a significant role in shaping the outcomes and the impact of the disease on this population.

\section{Conclusions}

A relatively low COVID-19-related morbidity and mortality were observed at 6 weeks from the start of the outbreak in our Region, one of the hardest hit areas in Europe. Measures to protect the most complex and vulnerable individuals in our society, including many of our ACHD patients, are crucial, until a vaccine will be developed. All ACHD centers must provide support and care for their patients in this difficult time, educating them on the precautions necessary to minimize risk and the impact of this pandemic.

\section{References}

1. Grasselli G, Zangrillo A, Zanella A, et al. Baseline characteristics and outcomes of 1591 patients infected with SARS-CoV-2 admitted to ICUs of the Lombardy Region, Italy. JAMA 2020 [Epub ahead of print].

2. Huang C, Wang Y, Li X, et al. Clinical features of patients infected with 2019 novel coronavirus in Wuhan, China. Lancet 2020;395:497-506.

3. Driggin E, Madhavan MV, Bikdeli B, et al. Cardiovascular considerations for patients, health care workers, and health systems during the coronavirus disease 2019 (COVID-19) pandemic. J Am Coll Cardiol 2020. [Epub ahead of print].

4. Zhou F, Yu T, Du R, et al. Clinical course and risk factors for mortality of adult inpatients with COVID-19 in Wuhan, China: a retrospective cohort study. Lancet 2020;0. [Epub ahead of print].

5. Stout KK, Daniels CJ, Aboulhosn JA, et al. 2018
AHA/ACC Guideline for the management of adults with congenital heart disease: executive summary: a report of the American College of Cardiology/American Heart Association Task Force on Clinical Practice Guidelines. J Am Coll Cardiol 2019;73:1494-563.

6. Tan W, Aboulhosn J. The cardiovascular burden of coronavirus disease 2019 (COVID-19) with a focus on congenital heart disease. Int J Cardiol 2020 [Epub ahead of print].

7. Elkind MSV, Harrington RA, Benjamin IJ. The role of the American Heart Association in the global COVID19 pandemic. Circulation 2020;141:e743-5.

8. Skulstad H, Cosyns B, Popescu BA, et al. COVID-19 pandemic and cardiac imaging: EACVI recommendations on precautions, indications, prioritization, and protection for patients and healthcare personnel. Eur Heart $\mathrm{J}$ Cardiovasc Imaging 2020 [Epub ahead of print].

9. Lakkireddy DR, Chung MK, Gopinathannair R, et al. Guidance for cardiac electrophysiology during the coronavirus (COVID-19) pandemic from the Heart Rhythm Society COVID-19 task force; Electrophysiology section of the American College of Cardiology; and the Electrocardiography and Arrhythmias Committee of the Council on Clinical Cardiology, American Heart Association. Circulation 2020 [Epub ahead of print].

10. Warnes CA, Liberthson R, Danielson GK, et al. Task force 1: the changing profile of congenital heart disease in adult life. J Am Coll Cardiol 2001;37:1170-5.

11. Wang B, Li R, Lu Z, Huang Y. Does comorbidity increase the risk of patients with COVID-19: evidence from metaanalysis. Aging 2020;12. [Epub ahead of print].

12. Organization WH. Rational use of personal protective equipment (PPE) for coronavirus disease (COVID-19): interim guidance, 19 March 2020. [Epub ahead of print].

13. Beeching NJ, Fletcher TE, Beadsworth MBJ. COVID19: testing times. BMJ 2020;369:m1403.

14. Tutarel O, Alonso-Gonzalez R, Montanaro C, et al. Infective endocarditis in adults with congenital heart disease remains a lethal disease. Heart $\mathrm{Br}$ Card Soc 2018;104:161-5.

15. Constantine A, Barradas-Pires A, Dimopoulos K. Modifiable risk factors in congenital heart disease: Education, transition, digital health and choice architecture. Eur J Prev Cardiol 2019:2047487319874146.

16. Wang K, Gheblawi M, Oudit GY. Angiotensin converting enzyme 2: a double-edged sword. Circulation 2020 [Epub ahead of print]..

17. Yang H, Bouma BJ, Dimopoulos K, et al. Non-vitamin $\mathrm{K}$ antagonist oral anticoagulants (NOACs) for thromboembolic prevention, are they safe in congenital heart disease? Results of a worldwide study. Int J Cardiol 2020;299:123-30.

18. Matchar DB, Jacobson A, Dolor R, et al. Effect of home testing of international normalized ratio on clinical events. N Engl J Med 2010;363:1608-20. 